\title{
Case Report: Intraarticular Synovial Sarcoma Causing Chronic Wrist Pain: A Rare Case Report
}

Farid Najdmazhar ${ }^{1}$ (D, Tina Shooshtarizadeh ${ }^{1}$, Seyed Matin Sadat Kiaei $^{1^{*}}$ (D)

1. Bone and Joint Reconstruction Research Center, Shafa Orthopedic Hospital, Iran University of Medical Sciences, Tehran, Iran.

Citation Najdmazhar F, Shooshtarizadeh T, Sadat Kiaei SM. Intraarticular Synovial Sarcoma Causing Chronic Wrist Pain: A Rare Case Report. Journal of Research in Orthopedic Science. 2020; 7(3):141-146. http://dx.doi.org/10.32598/JROSJ.7.3.704.1

dol $:$ : http://dx.doi.org/10.32598/JROSJ.7.3.704.1

\section{(c) (1) (8)}

Article info:

Received: 21 Apr 2020

Revised: 29 Apr 2020

Accepted: $05 \mathrm{Jul} 2020$

Available Online: 01 Aug 2020

Keywords:

Intraarticular, Synovial sarcoma, Wrist pain, Hand

\begin{abstract}
A B S T RA C T
Synovial Sarcoma (SS) is a rare and aggressive sarcoma that usually occurs around the knee joint; it rarely involves the hand. Joint involvement by SS is extremely rare. We report a case of wrist intraarticular SS presenting with chronic wrist pain. The patient underwent surgical excision alongside with local radiotherapy and chemotherapy. After 24 months of follow up, there was no recurrence or metastasis. Intraarticular SS should be in a differential diagnosis list of chronic wrist pain even though it is extremely rare.
\end{abstract}

\section{Introduction}

oft tissue sarcomas of the hand are rare and comprise only $1 \%$ of the adult sarcomas $[1,2]$. Synovial Sarcoma (SS) is a rare and aggressive sarcoma that usually involves lower extremities, with a predilection for the knee area; it rarely occurs in the hand [1]. Joint involvement by SS is extremely rare [2]. Intraarticular SS has been reported in the knee, elbow, and hip joints and the knee joint being the most common involved one [2]. This study reports an intraarticular SS of the wrist joint causing chronic wrist pain.

\section{Case Presentation}

A 56-year-old female has been referred to the hand clinic with severe pain and swelling over her right wrist. She suffered from pain and mild swelling since 5 years ago which increased over the last year.

The patient received different modality of treatments, including intraarticular corticosteroid injection and casting during that period with a possible diagnosis of dorsal wrist ganglion in other medical facilities before referring to our center.

Her systemic physical examination was normal. In the local examination, the wrist was moderately swol-

\section{* Corresponding Author:}

Seyed Matin Sadat Kiaei, PhD.

Address: Bone and Joint Reconstruction Research Center, Shafa Orthopedic Hospital, Iran University of Medical Sciences, Tehran, Iran. Phone: +98 (912) 989120716373

E-mail: matin.sk2@gmail.com 


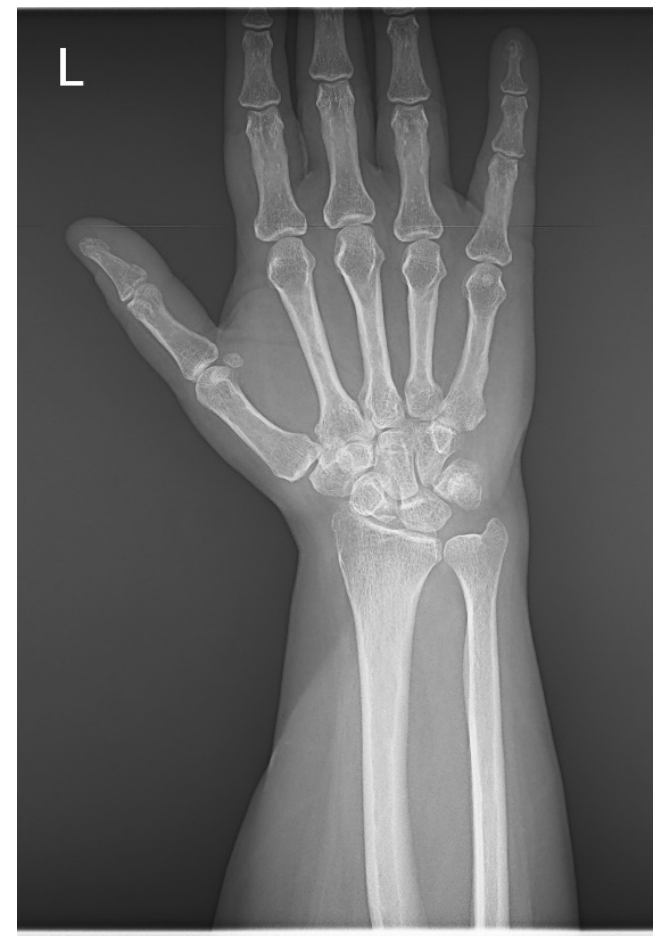

Figure 1. Normal plain radiographs of the wrist

len and severely tender in palpation of its dorsal aspect. The pain was exacerbated by cold exposure and the result of Hildreth's test was positive. The active range of motion was severely limited due to pain. The neurovascular assessment was normal.

Laboratory test results were within normal limits. Wrist radiography was reported normal (Figure 1). MRI revealed a dorsal intraarticular mass with an intermediate signal in T1 weighted and high signal in T2 weighted images reported as wrist ganglion by a radiologist (Figure 2 ). With the possible diagnosis of glomus tumor, we de-

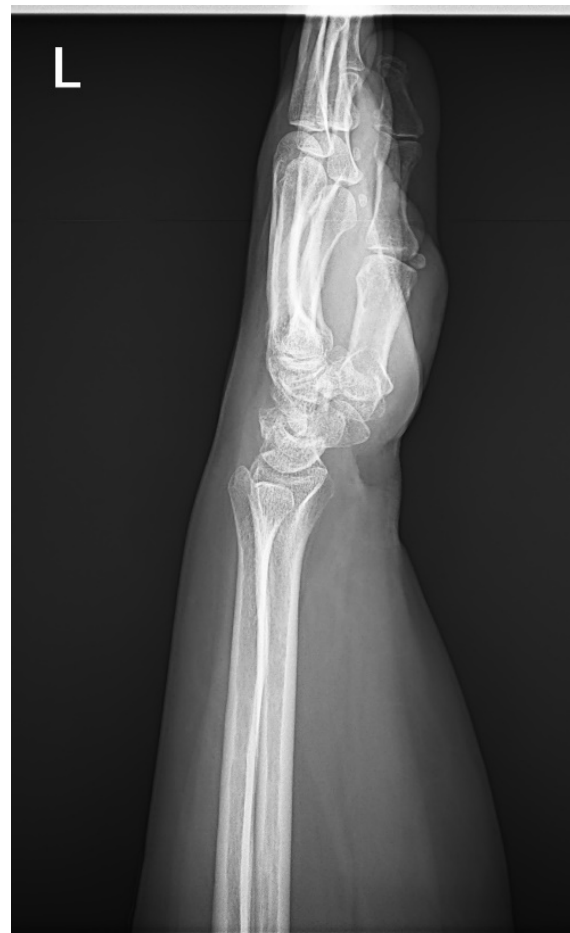

Orthopedic Science

cided to do the excisional biopsy. Through straight longitudinal incision between the $3^{\text {rd }}$ and $4^{\text {th }}$ compartments and after the opening of the extensor retinaculum, the extensor tendons were moved to radial and ulnar side to expose the wrist dorsal capsule that was found intact (Figure 3). Then, we excised the posterior capsule with the attached tumor which was a solid vascular one (Figure 4).

The size of the lesion was approximately $3 \times 2 \times 1 \mathrm{~cm}$. It consisted of grayish-white tiny nodules with rubbery consistency beneath the synovial membrane. The specimen was sent for histologic examination. Microscopi-

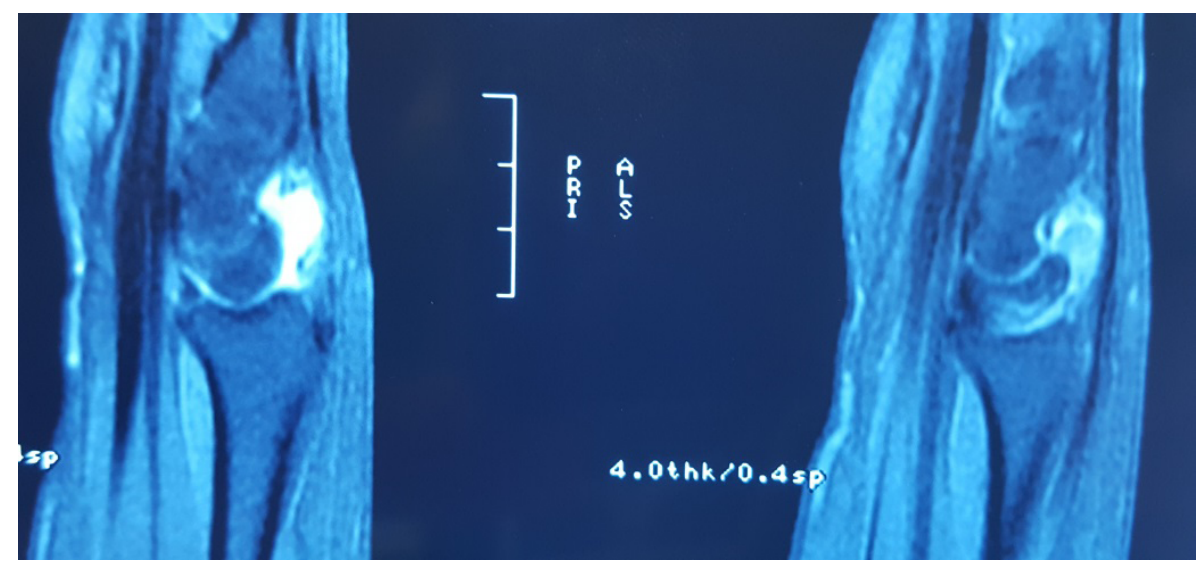

Orthopedic Science

Figure 2. Magnetic resonance imaging of the wrist depicting dorsal intraarticular mass with intermediate signal in T1 weighted and high signal in $\mathrm{T} 2$ weighted images 


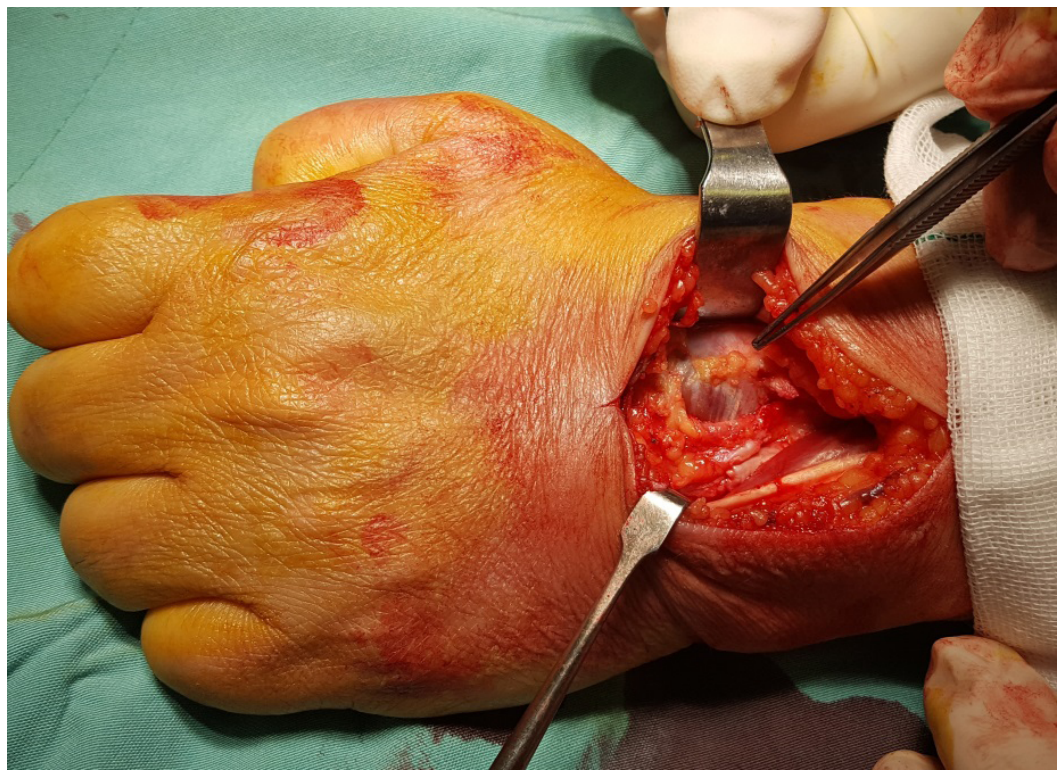

Figure 3. Intraoperative photograph, forceps pointing to the intact and bulging dorsal wrist capsule

Orthopedic Science

cally, the tumor was a hypercellular biphasic neoplasm, lined by synovium. It was composed of 2 types of tumor cells, including a solid proliferation of rather slender spindle cells of uniform morphology with scant amphiphilic cytoplasm and dark-staining nuclei and minor population of epithelioid cells embedded in the former population in small aggregates. The epithelioid cells were large oval cells with vesicular nuclei and abundant pale cytoplasm.

No necrosis was identified. Rare mitotic figures were noted. The immunohistochemical study revealed positive reactivity for Epithelial Membrane Antigen (EMA) and Transducing-Like Enhancer Protein1 (TLE1), but negative reactivity for Cytokeratin (CK). The final diagnosis was synovial sarcoma (Figure 5). Chest and abdominal CT scan alongside with whole-body bone scan were negative for metastasis. After discussing the diagnosis and the situation with our patient, we did the second operation, excising tumor bed, and resecting the incisional tract. The histological examination result for these tissues was free of tumor. Next, the patient was referred to the oncologist. She underwent local radiotherapy and systemic chemotherapy.

After 24 months, she had a painless wrist with an acceptable range of motion. Radiographs and MRI are negative for recurrence at present.

\section{Discussion}

Synovial Sarcoma (SS) is a rare and aggressive sarcoma that usually occurs in young adults between the ages of 15 and 40 years mostly involving lower extremities, with a predilection for the knee area $[1,3]$.

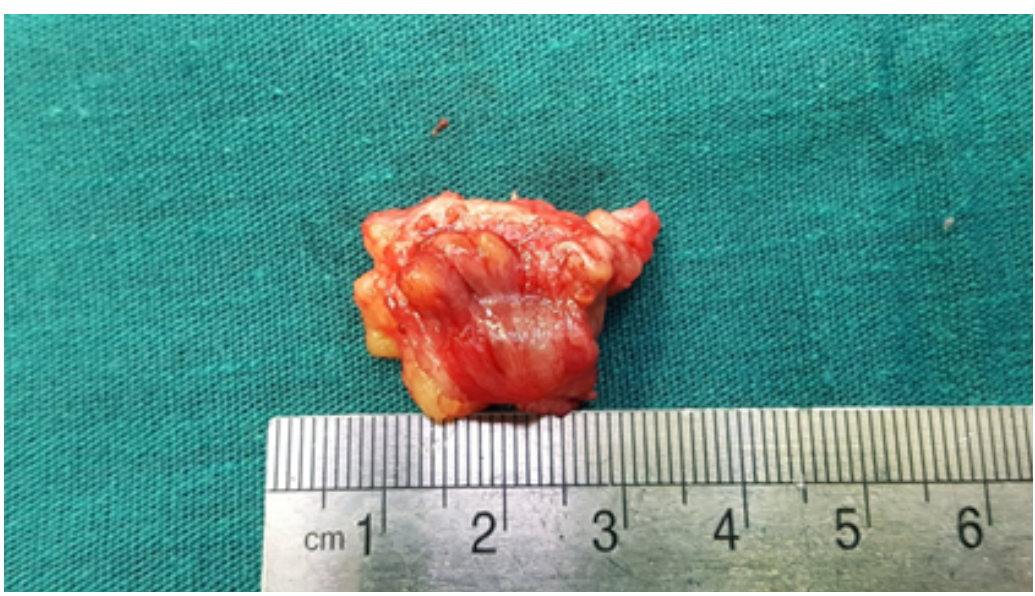

Figure 4. The excised tumor 
A

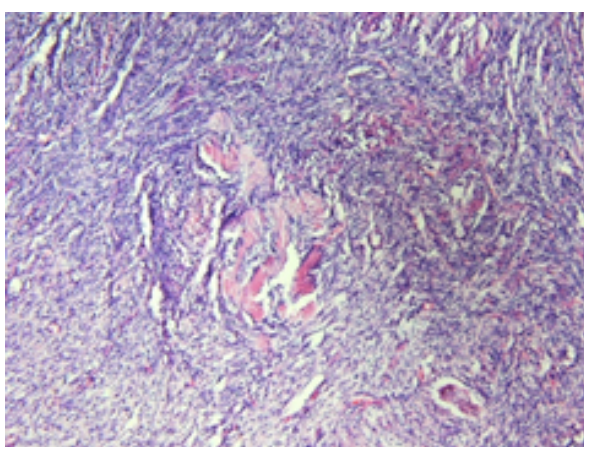

c

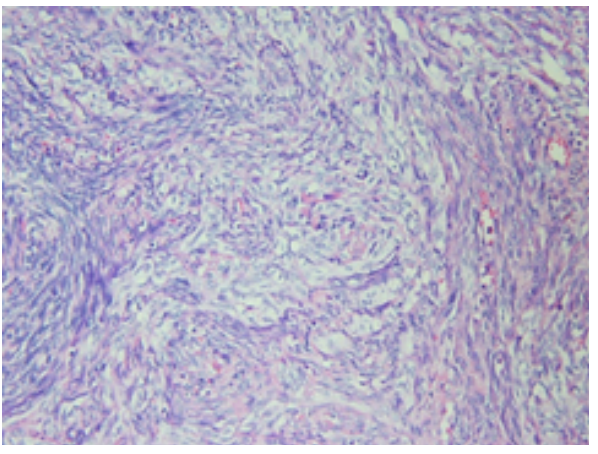

E

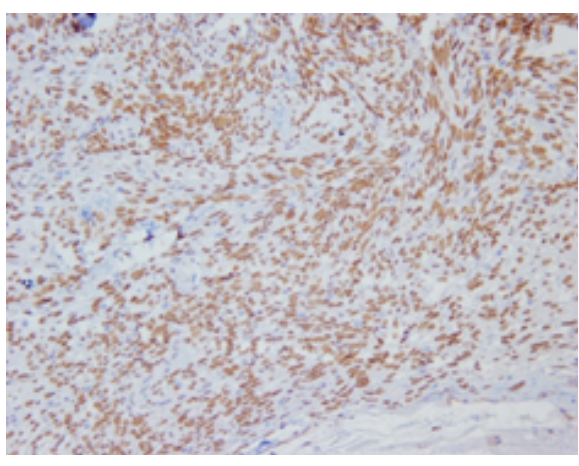

B

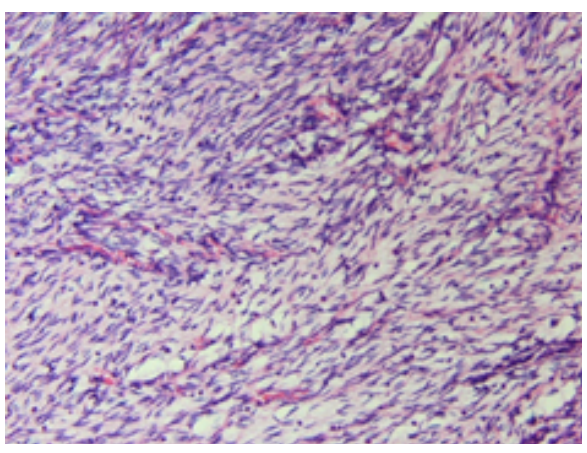

D

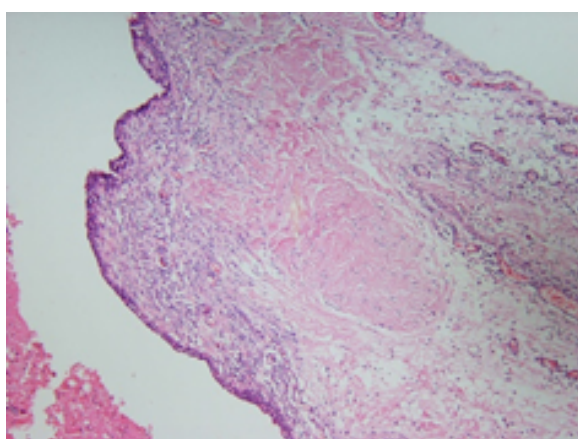

$\mathbf{F}$

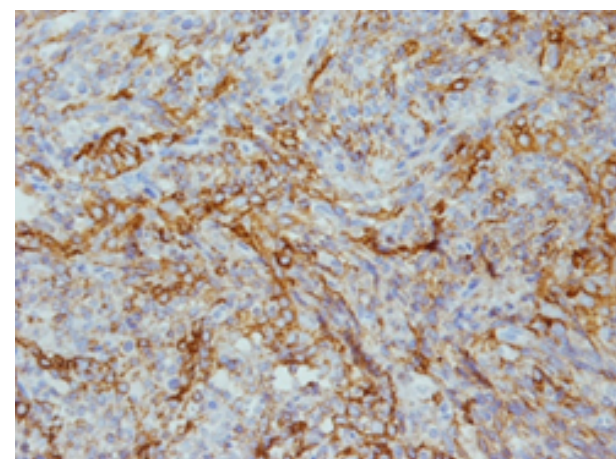

Orthopedic Science

Figure 5. Microscopic appearance of the neoplasm

A. Spindle cell proliferation of high cellularity in short fascicles (100 x magnification); B. Showing rather uniform slender nuclei (x 200 magnification); C. Nests of epithelial cells with clear cytoplasm (200 x magnification); D. Infiltration of subsynovial area by the neoplasm (100 x magnification); E. Strong nuclear TLE1 reactivity (200 x magnification); and F. EMA immunoreactivity (400 x magnification)

The origin of SS is unclear. It usually arises in synovium lacking organs and joint involvement is rare so it is thought that SS does not arise from synovial cells [4-6].

SS rarely occurs in the hand. The typical clinical presentation of synovial sarcoma of the hand is a slow-growing mass that rarely accompanies pain and tenderness.
Although SS has the highest incidence among intra-articular sarcomas [3], the extremely rare reported intra-articular SS is seen in the large joints of the lower extremity, especially the knee joint [4]. A comprehensive review of the English literature indicated that only 19 primary intraarticular synovial sarcomas have been reported [2]. To 
our knowledge, this case is the first one of the intraarticular SS of the wrist joint presented by chronic wrist pain.

The diagnosis of the intraarticular SS is misleading due to its rare occurrence. The reasons for a swollen painful wrist could be diverse and numerous. Although some MRI features, including multiloculated or lobulated septate cystic masses with heterogeneous septal manifestation, suggested for an intraarticular SS [2], this was not the case for our patient. According to the presenting case, a ganglion cyst can mimic the MRI manifestations of a wrist intraarticular SS.

Histologically, synovial sarcoma is a biphasic neoplasm with a specific chromosomal translocation $\mathrm{t}(\mathrm{X} ; 18)$. It is composed of two morphologically different types of cells: epithelial cells that can arrange in nests, cords, or glandular structures and spindle cells of rather uniform morphology. The neoplasm is classified as a biphasic or monophasic type depending on the relative proportion of each component. The poorly differentiated subtype is another form that behaves more aggressively. In the present case, the tumor was predominantly composed of spindle cells showing focal epithelial differentiation.

Most synovial sarcomas display immunoreactivity for EMA and CK and usually show diffuse and strong reactivity for TLE1. The present neoplasm was CK negative and the diagnosis was confirmed by TLE1 immunoreactivity.

The treatment of synovial sarcoma of hand is surgical excision with an acceptable margin which may be accompanied by radiotherapy and chemotherapy.

Unplanned excision of the soft tissue sarcoma of the hand is not uncommon due to the rare occurrence of this tumor. This issue can be true for intraarticular SS which is extremely rare but happened for our patient.

\section{Conclusions}

Intraarticular SS should be in a differential diagnosis list in a patient with chronic wrist pain although it is extremely rare.

\section{Ethical Considerations}

\section{Compliance with ethical guidelines}

All ethical principles are considered in this article. The participants were informed about the purpose of the research and its implementation stages; they were also assured about the confidentiality of their information; moreover, they were free to leave the study whenever they wished, and if desired, the research results would be available to them.

\section{Funding}

This research did not receive any grant from funding agencies in the public, commercial, or non-profit sectors.

\section{Authors' contributions}

All authors contributed in preparing this article.

\section{Conflict of interest}

The authors declared no conflict of interest.

\section{References:}

[1] Puhaindran ME, Athanasian EA. Double ray amputation for tumors of the hand. Clin Orthop Relat Res 2010; 468:29769. [DOI:10.1007/s11999-010-1389-2] [PMID] [PMCID]

[2] Friedman MV, Kyriakos M, Matava MJ, McDonald DJ, Jennings JW, Wessell DE. Intra-articular synovial sarcoma. Skeletal Radiol 2013; 42:859-67. [DOI:10.1007/s00256-0131589-4] [PMID]

[3] Helpert C, Davies AM, Evans M, Grimer RJ. Differential diagnosis of tumors and tumor-like lesions of the infrapatellar (Hoffa's) fat pad: Pictorial review with an emphasis on MR imaging. Eur Radiol. 2004; 14:2337e2346. [DOI:10.1007/ s00330-004-2491-1] [PMID]

[4] Bui-Mansfield LT, O’Brien SD. Magnetic resonance appearance of intra-articular synovial sarcoma: Case reports and review of the literature. J Comput Assist Tomogr. 2008; 32(4):640-4. [DOI:10.1097/RCT.0b013e31814cf96a] [PMID]

[5] Pradhan A, Cheung YC, Grimmer RJ, Peake D, Al-Muderis OA, Thomas JM, et al. Soft-tissue sarcomas of the hand: Oncological outcome and prognostic factors. Br J Bone Joint Surg. 2008; 90-B:209-14. [DOI:10.1302/0301620X.90B2.19601] [PMID]

[6] Lin PP, Guzel VB, Pisters PW, Zagars GK, Weber KL, Feig BW, et al. Surgical management of soft tissue sarcomas of the hand and foot. Cancer. 2002; 95:852-61. [DOI:10.1002/ cncr.10750] [PMID] 
This Page Intentionally Left Blank 